\title{
HOMOGENEOUS TOTALLY REAL SUBMANIFOLDS OF $S^{6}$
}

\author{
By
}

\author{
Katsuya Mashimo
}

It is well-known that the 6-dimensional sphere $S^{6}$ admits an almost complex structure. Among all submanifolds of almost complex manifold, there are two typical classes of submanifolds: namely the class of holomorphic submanifolds and the class of totally real submanifolds. By using a recent result of Harvey and Lawson [5], a cone over a 2-dimensional holomolphic (or a 3-dimensional totally real) submanifold of $S^{6}$ is a stable minimal submanifold of $R^{7}$.

On the existence of such submanifolds, a satisfying result on holomorphic submanifolds was obtained by R. Bryant [1], i.e., he proved that every compact Riemann surface can be realized as a holomorphic curve of $S^{6}$.

On the contrary, we do not have such a satisfying result on the existence of 3-dimensional totally real submanifolds of $S^{6}$. In this paper we classify 3-dimensional compact totally real submanifolds of $S^{6}$, which are obtained as orbits of closed subgroups of $G_{2}$.

\section{Cayley algebra and the exceptional simple Lie group $G_{2}$}

In this section we give a brief review on Cayley algebra and the exceptional simple Lie group $G_{2}$.

Let $\boldsymbol{H}$ be the skew field of all quaternions. Then the Cayley algebra $\boldsymbol{C a}$ over $\boldsymbol{R}$ is $\boldsymbol{C a}=\boldsymbol{H}+\boldsymbol{H}$ with the following multiplication:

$$
(q, r) \cdot(s, t)=(q s-\bar{t} r, t q+r \bar{s}), q, r, s, t \in \boldsymbol{H}
$$

where "-" means the conjugation in $\boldsymbol{H}$. We define a conjugation in $\boldsymbol{C a}$ by $\overline{(q, \boldsymbol{r})}$ $=(\bar{q},-\boldsymbol{r}), q, \boldsymbol{r} \in \boldsymbol{H}$, and an inner product $\langle$,$\rangle by$

$$
\langle x, y\rangle=(x \cdot \bar{y}+y \cdot \bar{x}) / 2, x, y \in \boldsymbol{C a} .
$$

Let $1, i, j, k$ be the standard basis of $\boldsymbol{H}$. Then $e_{0}=(1,0), e_{1}=(i, 0), e_{2}=(j, 0)$, $e_{3}=(k, 0), e_{4}=(0,1), e_{5}=(0, i), e_{6}=(0, j), e_{7}=(0, k)$ form an orthonormal basis of Ca. We put

$$
\boldsymbol{C a} \boldsymbol{a}_{0}=\{x \in \boldsymbol{C a} \mid x+\bar{x}=0\}=\sum_{j=1}^{7} \boldsymbol{R} e_{j}
$$

Received October 5, 1985. 
Then we have the following multiplication table

\begin{tabular}{|c|c|c|c|c|c|c|c|c|}
\hline \multirow{8}{*}{$e_{i} \cdot e_{j}=$} & $i / j$ & 1 & 2 & 3 & 4 & 5 & 6 & 7 \\
\hline & 1 & $-e_{0}$ & $e_{3}$ & $-e_{2}$ & $e_{5}$ & $-e_{4}$ & $-e_{7}$ & $e_{6}$ \\
\hline & 2 & $-e_{3}$ & $-e_{0}$ & $e_{1}$ & $e_{6}$ & $e_{7}$ & $-e_{4}$ & $-e_{5}$ \\
\hline & 3 & $e_{2}$ & $-e_{1}$ & $-e_{0}$ & $e_{7}$ & $-e_{6}$ & $e_{5}$ & $-e_{4}$ \\
\hline & 4 & $-e_{5}$ & $-e_{6}$ & $-e_{7}$ & $-e_{0}$ & $e_{1}$ & $e_{2}$ & $e_{3}$ \\
\hline & 5 & $e_{4}$ & $-e_{7}$ & $e_{6}$ & $-e_{1}$ & $-e_{0}$ & $-e_{3}$ & $e_{2}$ \\
\hline & 6 & $e_{7}$ & $e_{4}$ & $-e_{5}$ & $-e_{2}$ & $e_{3}$ & $-e_{0}$ & $-e_{1}$ \\
\hline & 7 & $-e_{6}$ & $e_{5}$ & $e_{4}$ & $-e_{3}$ & $-e_{2}$ & $e_{1}$ & $-e_{0}$ \\
\hline
\end{tabular}

The Cayley algebra $\boldsymbol{C a}$ is neither commutative nor associative. But we have the following

Lemma 1.1. (1) If $x, y \in \boldsymbol{C} \boldsymbol{a}_{0}$, then $x \cdot y=-y \cdot x$.

(2) For any $x, y, z \in \boldsymbol{C a}$,

$$
\begin{aligned}
& \bar{x} \cdot(x \cdot y)=(\bar{x} \cdot x) \cdot y, \\
& \langle x \cdot y, x \cdot z\rangle=\langle x, x\rangle\langle y, z\rangle .
\end{aligned}
$$

(3) Let $x, y, z \boldsymbol{C a}$ be mutually orthogonal unit vectors. Then

$$
x \cdot(y \cdot z)=y \cdot(z \cdot x)=z \cdot(x \cdot y) .
$$

For the proof, we refer to [4].

It is well-known that the group of all automorphisms of $\boldsymbol{C a}$ is a compact connected simple Lie group of type $\mathbb{S}_{2}([4])$. So we denote it by $G_{2}$. Then $G_{2}$ leaves the vector $e_{0}$ and the subspace $\boldsymbol{C} \boldsymbol{a}_{0}$ invariant. Furthermore $G_{2}$ leaves the inner product $\langle$,$\rangle invariant. So we may regard G_{2}$ as a subgroup of $S O(7)=S O\left(\boldsymbol{C a}_{0}\right)$.

Lemma 1.2. Let $v_{1}, v_{2}, v_{3}$ be mutually orthogonal unit vectors in $\boldsymbol{C a}_{0}$ with $\left\langle v_{1}\right.$. $\left.v_{2}, v_{3}\right\rangle=0$. Then there exists a (unique) automorphism $g$ of $\boldsymbol{C a}$ such that $g\left(e_{i}\right)=v_{i}$, $i=1,2,3$.

For the proof of Lemma 1.2, we refer to [5].

Let $G_{i j}, 1 \leqq i \neq j \leqq 7$ be the skew symmetric transformation on $\boldsymbol{C a}_{0}$ defined by

$$
G_{i j}\left(e_{k}\right)=\left\{\begin{aligned}
e_{i}, & \text { if } k=j, \\
-e_{j}, & \text { if } k=i, \\
0, & \text { otherwise. }
\end{aligned}\right.
$$

Then the Lie algebra $\mathbb{B}_{2}$ of $G_{2}$ is spanned by the following vectors in the Lie algebra $\mathfrak{s}_{0}(7)$ of $S O(7)$.

$$
a G_{23}+b G_{45}+c G_{76},
$$




$$
\begin{aligned}
& a G_{31}+b G_{46}+c G_{57}, \\
& a G_{12}+b G_{47}+c G_{65}, \\
& a G_{51}+b G_{73}+c G_{62}, \\
& a G_{14}+b G_{72}+c G_{36}, \\
& a G_{17}+b G_{24}+c G_{53}, \\
& a G_{61}+b G_{34}+c G_{25}, \quad a+b+c=0, a, b, c \in \boldsymbol{R} .
\end{aligned}
$$

\section{$\S 2$. Stability of cones over totally real submanifolds}

In this section we briefly summalize the results of Harvey and Lawson [5] and study the cones over 3-dimensional totally real submanifolds of $S^{6}$.

Let $M$ be an n-dimensional Riemannian manifold and let $G_{p}(M)$ be the bundle of p-planes of $M$. Then we can regard $G_{p}(M)$ as a subset of the p-th exterior power $\Lambda^{p}(M)$ of the tangent bundle of $M$ in a natural manner. Then any exterior $\mathrm{p}$-form on $M$ can be considered as a function on $G_{p}(M)$. The comass of an exterior p-form $\phi$ is defined by

$$
\|\phi\|^{*}=\sup _{\xi \in G_{p}(M)} \phi(\xi) .
$$

Assuming $\|\phi\|^{*}=1$, we put

$$
G(\phi)=\left\{\xi \in G_{p}(M) \mid \phi(\xi)=1\right\} .
$$

A p-dimensional, oriented $C^{1}$-submanifold $S$ of $M$ is called a $\phi$-manifold if the oriented p-plane $T_{x}(S)$ is contained in $G(\phi)$ for all $x \in S$.

THEOREM 2.1 (Harvey and Lawson, [5]). Let $\phi$ be a closed $\mathrm{p}$-form with $\|\phi\|^{*}=1$ and $S$ be a compact $\phi$-manifold. Then $\operatorname{Vol}(S) \leqq \operatorname{Vol}\left(S^{\prime}\right)$ for any compact submanifold $S$ ' of $M$ which is homologous to $S$.

In [5], Harvey and Lawson considered 2 calibrations on $\boldsymbol{C} \boldsymbol{a}_{0}$. Let $\boldsymbol{\phi}$ be a trilinear function on $\boldsymbol{C a}_{0}$ defined by

$$
\phi(x, y, z)=\langle x, y \cdot z\rangle, x, y, z \in \boldsymbol{C} a_{0} .
$$

Then by Lemma 1.1 it is easily seen that $\phi$ is a closed 3 -form on $\boldsymbol{C a}_{0}$. Furthermore $\phi$ has comass one ([5]). We fix an orientation on $\boldsymbol{C} \boldsymbol{a}_{0}$ such that $e_{1}, e_{2}, \cdots, e_{7}$ is an oriented basis and let $*$ be the Hodge star operator. Then $* \phi$ is a closed 4 -form and also has comass one ([5]). A $\phi$-manifold is called an associative submanifold and a ${ }^{*} \phi$-manifold is called a coassociative submanifold.

Let $S^{6}$ be the unit sphere in $\boldsymbol{C a}_{0}$ centered at the origin. Then $S^{6}$ has an almost complex structure $J$ defined by 


$$
J_{p}(X)=p \cdot X, \quad X \in T_{p}\left(S^{6}\right) .
$$

From the definition, $J$ is preserved by $G_{2}$. A submanfold $M$ of $S^{6}$ is called a holomorphic submanifold (resp. totally real submanifold) if $J\left(T_{p}(M)\right)=T_{p}(M)$ (resp. $J\left(T_{p}\right.$ $(M))$ is contained in the normal space $N_{p}(M)$ ) for any $p \in M$. We denote by $C M$ the cone over $M$.

TheOREM 2.2. Let $M$ be a 2-dimensional submanifold of $S^{6}$. Then $M$ is a holomorphic submanifold of $S^{6}$ if and only if $C M-\{0\}$ is an associative submanifold of $\boldsymbol{C a}_{0}$.

The proof of the above Theorem is easy so that we omit it.

TheOREM 2.3. Let $M$ be a 3-dimensional submanifold of $S^{6}$. Then $M$ is $a$ totally real submanifold of $S^{6}$ if and only if $C M-\{0\}$ is a coassociative submanifold of $\boldsymbol{C} \boldsymbol{a}_{0}$.

It is well-known that a submanifold $M$ of $S^{N}$ is a minimal submanifold if and only if $C M-\{0\}$ is a minimal submanifold of $\boldsymbol{R}^{N+1}$. Thus we have the following

Corollary 2.4 (Ejiri, [3]). Any 3-dimensional totally real submaifold of $S_{1}^{6}$ is a minimal submanifold.

Proof of Theorem 2.3. "if" part. Let $p$ be a point of $M$, and put $p=u_{4}$. Let $u_{5}, u_{6}, u_{7}$ be an orthonormal basis of $T_{p}(M)$ and $u_{1}, u_{2}, u_{3}$ be an orthonormal basis of the normal space $N_{p}(M)$ of $M$ at $p$ in $S^{6}$ such that $u_{1}, u_{2}, \cdots, u_{7}$ is oriented. Since ${ }^{*} \phi\left(u_{4} \wedge u_{5} \wedge u_{6} \wedge u_{7}\right)=\dot{\phi}\left(u_{1} \wedge u_{2} \wedge u_{3}\right)= \pm 1$, we get $u_{1} \cdot u_{2}= \pm u_{3}, u_{2} \cdot u_{3}= \pm u_{1}$ and $u_{3} \cdot u_{1}= \pm u_{2}$. By Lemma 1.1, we get

$$
\begin{aligned}
\left\langle J\left(u_{1}\right), u_{1}\right\rangle & =\left\langle u_{4} \cdot u_{1}, u_{1}\right\rangle=-\left\langle u_{1} \cdot u_{4}, u_{1}\right\rangle \\
& =-\left\langle u_{4}, \bar{u}_{1}, u\right\rangle=0, \\
\left\langle J\left(u_{1}\right), u_{2}\right\rangle & =\left\langle u_{4} \cdot u_{1}, u_{2}\right\rangle=-\left\langle u_{1} \cdot u_{4}, u_{2}\right\rangle \\
& =-\left\langle u_{4}, \bar{u}_{1} \cdot u_{2}\right\rangle=0, \\
\left\langle J\left(u_{1}\right), u_{3}\right\rangle & =\left\langle u_{4} \cdot u_{1}, u_{3}\right\rangle=-\left\langle u_{1} \cdot u_{4}, u_{3}\right\rangle \\
& =-\left\langle u_{4}, \bar{u}_{1} \cdot u_{3}\right\rangle=0,
\end{aligned}
$$

i.e., $J\left(u_{1}\right)$ is contained in $T_{p}(M)$. Similarly we see that $J\left(u_{2}\right)$ and $J\left(u_{3}\right)$ are contained in $T_{p}(M)$. Since $J$ is non-singular and $\operatorname{dim} N_{p}(M)=\operatorname{dim} T_{p}(M)$, we get $J\left(T_{p}(M)\right)=$ $N_{p}(M)$.

" only if" part. Let $u_{5}, u_{6}, u_{7}$ be an orthonormal basis of $T_{p}(M)$. By a simple calculation, $\left(\nabla_{u_{5}} J\right)\left(u_{6}\right)$ is equal to the tangential part of $u_{5} \cdot u_{6}$ to $S^{6}$, where $\nabla$ is the 
covariant derivative of $S^{6}$. Since $M$ is a totally real submanifold, $u_{5} \cdot u_{6}$ is normal to $p$. So $\left(\nabla_{u_{5}} J\right)\left(u_{6}\right)=u_{5} \cdot u_{6}$. In [3], Ejiri proved that $\left(\nabla_{u_{5}} J\right)\left(u_{6}\right)$ is normal to $M$. By Lemma 1. 1 (ii), $u_{5} \cdot u_{6}$ is normal to $p \cdot u_{5}$ and $p \cdot u_{6}$. Since $N_{p}(M)$ is of dimension 3 and $u_{5} \cdot u_{6}, p \cdot u_{7}$ are unit vectors, $u_{5} \cdot u_{6}= \pm p \cdot u_{7}$. Similarly $u_{6} \cdot u_{7}= \pm p \cdot u_{5}$ and $u_{7} \cdot u_{5}= \pm p \cdot u_{6}$. Then it is easily seen that $p \cdot u_{5}, p \cdot u_{6}, p \cdot u_{7}$ form an orthonormal basis of $T_{p}(M)$. Thus by Lemma 1.1 we obtain

$$
\begin{aligned}
{ }^{*} \phi\left(p \wedge u_{5} \wedge u_{6} \wedge u_{7}\right) & = \pm \phi\left(p \cdot u_{5} \wedge p \cdot u_{6} \wedge p \cdot u_{7}\right) \\
& = \pm<p \cdot u_{5},\left(p \cdot u_{6}\right) \cdot\left(p \cdot u_{7}\right)>= \pm 1 .
\end{aligned}
$$

Therefore $C M-\{0\}$ is a coassociative submanifold.

Q.E.D.

LEMMA 2.5. Let $M, M$ be two totally real submanifolds of constant curvature $1 / 16$ in $S_{1}^{6}$. Then there exists $g \in G_{2}$ such that $g(M)=M$.

Proof. In [3], Ejiri proved that the normal connection $\nabla^{\perp}$ of a totally real submanifold $M$ of $S_{1}^{6}$ is

$$
\nabla^{\perp} J Y=X \cdot Y+J\left(\nabla_{X} Y\right), X, Y \in T M .
$$

Furthermore he proved that if $M$ is a space of constant curvature then there exists a local orthonormal frame field $e_{1}, e_{2}, e_{3}$ such that

$$
\begin{aligned}
& \alpha\left(e_{1}, e_{1}\right)=15^{1 / 2} J e_{1} / 2, \\
& \alpha\left(e_{2}, e_{2}\right)=\left(-15^{1 / 2} J e_{1}+10^{1 / 2} J e_{2}\right) / 4, \\
& \alpha\left(e_{3}, e_{3}\right)=\left(-5^{1 / 5} J e_{1}-10^{1 / 2} J e_{2}\right) / 4, \\
& \alpha\left(e_{1}, e_{2}\right)=-5^{1 / 2} J e_{2}, \\
& \alpha\left(e_{2}, e_{3}\right)=-10^{1 / 2} J e_{3} / 4, \\
& \alpha\left(e_{1}, e_{3}\right)=-5^{1 / 2} J e_{3} / 4, \\
& \nabla_{e_{i}} e_{i}=0, i=1,2,3, \\
& \nabla_{e_{1}} e_{2}=-\nabla_{e_{2}} e_{1}=-e_{3} / 4, \\
& \nabla_{e_{2}} e_{3}=-\nabla_{e_{3}} e_{1}=-e_{1} / 4, \\
& \nabla_{e_{3}} e_{1}=-\nabla_{e_{1}} e_{3}=-e_{2} / 4, \\
& J\left(e_{1}, e_{2}\right)=e_{3}, J\left(e_{2}, e_{3}\right)=e_{1}, J\left(e_{3}, e_{1}\right)=e_{2} .
\end{aligned}
$$

where $\alpha$ is the second fundamental form of $M$.

Take a point $p \in M$ and a point $p^{\prime} \in M^{\prime}$. Let $e_{1}, e_{2}, e_{3}$ be an orthonormal frame of $M$ at $p$ and $e_{1}^{\prime}, e_{2}^{\prime}, e_{3}^{\prime}$, be an orthonormal frame of $M$ at $p^{\prime}$ with the above properties. Then by a well-known rigidity theorem there exists a rigid motion 
$\sigma \in 0(7)$ such that $\sigma(p)=p^{\prime}, \sigma\left(e_{i}\right)=e_{i}^{\prime}, i=1,2,3$. Since $\sigma$ preserves the second fundamental form $\alpha, \sigma\left(J e_{i}\right)=J e_{i}^{\prime}, i=1,2,3$. Put $v_{1}=e_{2} \cdot e_{3}, v_{2}=-J\left(e_{3}\right), v_{3}=e_{3} \cdot e_{1}, v_{4}=p$, $v_{5}=e_{1}, v_{6}=e_{3}$ and $v_{7}=e_{2}$. Define $v_{1}^{\prime}, \cdots, v_{7}^{\prime}$ in a similar manner. Then by Lemma 1.1 , it is easily seen that $v_{1}, \cdots, v_{7}$ and $v_{1}^{\prime}, \cdots, v_{7}^{\prime}$ satisfy the same table of multiplication (1.1), i.e., $\sigma$ is contained in $G_{2}$.

Q.E.D.

\section{§3. 3-dimensional closed subgroups of $\boldsymbol{G}_{2}$}

Let $\mathbb{S}$ be a compact simple Lie algebra and $t$ be a maximal abelian subalgebra of $\mathbb{B}$. Let $\mathfrak{l}$ be a complex simple 3 -dimensional subalgebra of $\mathbb{S}^{C}$. Then there exists a basis $H, X_{+}, X_{-}$of $\mathfrak{l}$ such that

$$
\left[H, X_{+}\right]=2 X_{+},\left[H, X_{-}\right]=-2 X_{-},\left[X_{+}, X_{-}\right]=H .
$$

We may assume that $H$ is contained in $t^{c}$, in fact in $(-1)^{1 / 2} t^{c}$. Hence $\alpha(H)$ is a real number for every root $\alpha$ of ${ }^{C} C$ with respect to $t^{C}$. Furthermore $\alpha(H)=0,1$ or 2 if $\alpha$ is a simple root [2, p. 166]. The weighted Dynkin diagram with weight $\alpha(H)$ added to each vertex $\alpha$ of the Dynkin diagram of $\mathbb{S}^{C}$ is called the characteristic diagram of $\mathfrak{l}$. Let $\mathfrak{l}$ and $\mathfrak{l}$ ' be 3 -dimensional simple Lie subalgebras of $\mathbb{S}^{\boldsymbol{C}}$. Then $\mathfrak{l}$ and $\mathfrak{l}$ are mutually conjugate if and only if $\mathfrak{l}$ and $\mathfrak{l}$ ' have the same characteristic diagrams.

Mal'cev [7] classified the 3-dimensional complex simple subalgebras of $\mathbb{G}_{2} \boldsymbol{C}$. From his classification, $\mathbb{G}_{2}{ }^{C}$ has 4 types of 3 -dimensional simple subalgebras as follows.

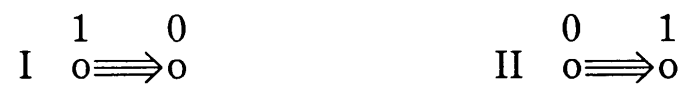

$$
\begin{aligned}
& \text { III } \stackrel{2}{\mathrm{O} \Longrightarrow \mathrm{0}} \stackrel{0}{\Rightarrow} \mathrm{IV} \stackrel{2}{\mathrm{0}} \Longrightarrow \mathrm{O}
\end{aligned}
$$

Let $\mathfrak{l}$ be a 3-dimensional simple subalgebra of $\mathbb{S}_{2}$. Then the complexification $\mathfrak{l}^{\boldsymbol{C}}$ of $\mathfrak{l}$ in $\mathbb{B}_{2}{ }^{\boldsymbol{C}}$ corresponds to one of the above 4 characteristic diagrams. As a special case of a Theorem of Siebenthal [[8], p. 252), we have the folowing.

LEMMA 3.1. Let $\mathfrak{l}$ and $\mathfrak{l}^{\prime}$ be 3-dimensional simple subalgebras of $\mathbb{S}_{2}$. If $\mathfrak{l}^{\mathrm{C}}$ and $\mathfrak{l}^{\prime C}$ correspond to the characteristic diagram $\stackrel{2}{0} \Longrightarrow 0,2$, then $\mathfrak{l}$ and $\mathfrak{l}$ ' are conjugate in $\mathrm{BS}_{2}$.

Similarly we can prove

Lemma 3.2. Let $\mathfrak{l}$ and $\mathfrak{l}$ ' be 3-dimensional simple subalgebras of $\mathbb{S}_{2}$. If ${ }_{\mathfrak{l}}$ C and 
$\mathfrak{l}^{\prime} \boldsymbol{C}$ correspond to the characteristic diagram $\stackrel{1}{\mathrm{0}} \Longrightarrow \mathrm{0}$ or $\stackrel{0}{0} \Longrightarrow \stackrel{1}{\Rightarrow}$, then $\mathfrak{l}$ and $\mathfrak{l}$, are conjugate in $\mathbb{B}_{2}$.

Now we give here an example of a basis $X_{1}, X_{2}, X_{3}$ of $\mathfrak{l}$ with $\left[X_{1}, X_{2}\right]=2 X_{3}$, $\left[X_{2}, X_{3}\right]=2 X_{1},\left[X_{3}, X_{1}\right]=2 X_{2}$. If $\mathfrak{l}$ corresponds to the characteristic diagram $\mathrm{I}$, then

$$
\begin{aligned}
& X_{1}=-G_{45}+G_{76}, \\
& X_{2}=-G_{46}+G_{57}, \\
& X_{3}=-G_{47}+G_{65} .
\end{aligned}
$$

If $\mathfrak{l}$ corresponds to the characteristic diagram II, then

$$
\begin{aligned}
& X_{1}=-2 G_{23}+G_{45}+G_{76}, \\
& X_{2}=-2 G_{31}+G_{46}+G_{57}, \\
& X_{3}=-2 G_{12}+G_{47}+G_{65} .
\end{aligned}
$$

If $\mathfrak{l}$ corresponds to the characteristic diagram IV, then

$$
\begin{aligned}
& X_{1}=4 G_{32}+2 G_{54}-6 G_{76}, \\
& X_{2}=6^{1 / 2}\left(G_{37}+G_{26}-2 G_{15}\right)+10^{1 / 2}\left(G_{42}-G_{35}\right), \\
& X_{3}=6^{1 / 2}\left(G_{63}+G_{27}-2 G_{41}\right)+10^{1 / 2}\left(G_{25}-G_{34}\right) .
\end{aligned}
$$

LEMMA 3.3. Let $\mathfrak{l}$ be a 3-dimensional simple subalgebra of $\mathbb{S}_{2}$. If $\mathfrak{l}^{\boldsymbol{C}}$ corre$2 \quad 0$

sponds to the characteristic diagram $\mathrm{0} \Longrightarrow \mathrm{O}$, then $\mathfrak{l}$ is spanned by the following basis $X_{1}, X_{2}, X_{3}$ for some $\theta$ :

$$
\begin{aligned}
& X_{1}=-2 G_{21}-2 G_{65}, \\
& X_{2}=-2 \cos \theta\left(G_{32}+G_{76}\right)-2 \sin \theta\left(G_{72}+G_{63}\right), \\
& X_{3}=-2 \cos \theta\left(G_{31}+G_{75}\right)-2 \sin \theta\left(G_{53}+G_{71}\right) .
\end{aligned}
$$

Proof. A simple computation shows that $\mathfrak{l}^{C}$ is conjugate to the Lie subalgebra spanned by

$$
\begin{aligned}
& H=2(-1)^{1 / 2}\left(G_{21}+G_{65}\right), \\
& X_{+}=-2\left(G_{32}+G_{76}\right)+2(-1)^{1 / 2}\left(G_{31}+G_{75}\right), \\
& X_{-}=2\left(G_{32}+G_{76}\right)+2(-1)^{1 / 2}\left(G_{31}+G_{75}\right) .
\end{aligned}
$$

Hence it is easily seen that $\sum_{i=1}^{3} \boldsymbol{R} e_{i}, \boldsymbol{R} e_{4}, \sum_{i=5}^{7} \boldsymbol{R} e_{i}$ are invariant irreducible components of $\boldsymbol{C a}_{0}^{\boldsymbol{C}}$ under the action of the subalgebra spanned by $H, X_{+}$and $X_{-}$ defined by (3.10), Therefore $\boldsymbol{C} \boldsymbol{a}_{0}$ has 2 invariant irreducible components $V_{1}, V_{2}$ 
of dimension 3 and an invariant irreducible component $V_{0}$ of dimension 1 under the action of $\mathfrak{l}$. Let $L$ be the Lie subgroup of $G_{2}$ generated by $\mathfrak{l}$. Remark that $L$ is isomorphic to $S O(3)$ and the actions of $L$ on $V_{1}$ and $V_{2}$ are equivalent to the standard action of $S O(3)$ on $\boldsymbol{R}^{3}$. Let $v_{4}$ be a unit vector in $V_{0}$. Take a one parameter subgroup $K$ in $L$. Then there are determined (up to sign) unit vectors $v_{1}$ in $V_{1}$ and $v_{5}$ in $V_{2}$. Since $v_{1} \cdot v_{4}$ is also a $\mathrm{K}$-fixed vector and is normal to $v_{1}$ and $v_{4}, v_{1} \cdot v_{4}$ is equal to $v_{5}$ or $-v_{5}$. By a change of sign (if necessary) we have $v_{1}$. $v_{4}=v_{5}$. Let $v_{2}$ be a unit vector in $V_{1}$ which is orthogonal to $v_{1}$ and $K^{\prime}$ be the isotropy subgroup at $v_{2}$. Then by a similar argument, we can choose a unit vector $v_{6}$ in $V_{2}$ such that $v_{2} \cdot v_{4}=v_{6}$. Put $v_{3}=v_{1} \cdot v_{2}$ and $v_{7}=v_{3} \cdot v_{4}$. Then by Lemma 1.2, there exists an automorphism $g$ of $\boldsymbol{C a}$ such that $g\left(e_{i}\right)=v_{i}$ for $i=1,2,4$. Since $g$ is an automorphism of $\boldsymbol{C a}$, we have $g\left(e_{3}\right)=v_{3}, g\left(e_{5}\right)=v_{5}, g\left(e_{6}\right)=v_{6}$ and $g\left(e_{7}\right)=v_{7}$. Hence $v_{1}, v_{2}, \cdots, v_{7}$ satisfy the same multiplication table (1.1) as $e_{1}, e_{2}, \cdots, e_{7}$. Let $v_{3}$, be a unit vector in $V_{1}$ which is orthogonal to $v_{1}$ and $v_{2}$. Then $v_{3}^{\prime}$ is of the form

$$
v_{3}^{\prime}=(\cos \theta) v_{3}+(\sin \theta) v_{7} .
$$

Take a suitable basis $X_{1}, X_{2}, X_{3}$ of $\mathfrak{l}$. Then the restrictions of $X_{1}, X_{2}$ and $X_{3}$ to $V_{1}$ are represented by the following matrices with respect to the basis $v_{1}, v_{2}$ and $v_{3}{ }^{\prime}$ :

$$
X_{1}=\left(\begin{array}{rrr}
0 & -2 & 0 \\
2 & 0 & 0 \\
0 & 0 & 0
\end{array}\right), \quad X_{2}=\left(\begin{array}{rrr}
0 & 0 & 0 \\
0 & 0 & -2 \\
0 & 2 & 0
\end{array}\right), \quad X_{3}=\left(\begin{array}{rrr}
0 & 0 & -2 \\
0 & 0 & 0 \\
2 & 0 & 0
\end{array}\right)
$$

Put $v_{7}^{\prime}=v_{4} \cdot v_{3}=-(\sin \theta) v_{3}+(\cos \theta) v_{7}$. Then $v_{5}, v_{6}$ and $v_{7}^{\prime}$, form an orthonormal basis of $V_{2}$. Since $X_{1}$ is contained in $\mathbb{B}_{2}$,

$$
\begin{aligned}
X_{1}\left(v_{5}\right) & =X_{1}\left(v_{1} \cdot v_{4}\right)=X_{1}\left(v_{1}\right) \cdot v_{4}+v_{1} \cdot X_{1}\left(v_{4}\right) \\
& =v_{1} \cdot X_{1}\left(v_{4}\right)=-2 v_{6} .
\end{aligned}
$$

By similar calculations, we get the representations of $X_{1}, X_{2}$ and $X_{3}$ restricted to $V_{2}$ with respect to $v_{5}, v_{6}$ and $v_{7}$. They are of the same form as $X_{1}, X_{2}$ and $X_{3}$ as above. Express $X_{1}, X_{2}$ and $X_{3}$ with respect to $v_{1}, \cdots, v_{7}$. Then we see that $\mathfrak{l}$ is conjugate to the subalgbra spanned by the following basis

$$
\begin{aligned}
& X_{1}=-2 G_{21}-2 G_{65}, \\
& X_{2}=-2 \cos \theta\left(G_{32}+G_{76}\right)-2 \sin \theta\left(G_{72}+G_{63}\right), \\
& X_{3}=-2 \cos \theta\left(G_{31}+G_{75}\right)-2 \sin \theta\left(G_{53}+G_{71}\right) .
\end{aligned}
$$




\section{$\S 4$. Homogeneous totally real submanifolds of $S^{6}$}

In this section we classify 3-dimensional compact homogeneous totally real submanifolds of $S^{6}$, which are obtained as orbits of closed subgroups of $G_{2}$.

First we study one by one the 4 types of subgroups which are generated by subalgebras listed in $\S 3$. In some cases it is convenient for us to find all orbits which are 3-dimensional minimal submanifolds of $S_{1}^{6}$, since a 3-dimensional totally real submanifold of $S_{1}^{6}$ is a minimal submanifold by Corollary 2.4.

CASE I. $\boldsymbol{R} e_{1}, \boldsymbol{R} e_{2}, \boldsymbol{R} e_{3}$ and $\sum_{j=4}^{7} \boldsymbol{R} e_{j}$ are irreducible invariant subspaces so that each orbit is a small sphere or a great sphere. Therefore the orbit we are looking for is a trivial one.

CASE II. This case was studied by Harvey and Lawson [5].

THEOREM 4.1. Let $L$ be the subgroup of $G_{2}$ generated by the subalgebra spanned by $X_{1}, X_{2}$ and $X_{3}$ defined by (3.7). Then there exists exactly one orbit which is $a$ 3-dimensional totally real submanifold of $S^{6}$. It is the orbit through $\left(5^{1 / 2} / 3\right) e_{1}+(2 / 3)$ $e_{5}$, which we denote by $M_{1}$.

CASE III. For this case we have the following

THEOREM 4.2. Let $L_{\theta}$ be the subgroup of $G_{2}$ generated by the subalgebra spanned by $X_{1}, X_{2}$ and $X_{3}$ defined by (3.5). Then there exists exactly one orbit under $L_{\theta}$ which is a 3-dimensional totally real submanifold of $S^{6}$. It is the orbit through $\left(2^{1 / 2} / 2\right)\left(e_{2}+e_{5}\right)$, which we denote by $M_{2}$.

Proof. In this case, $L_{\theta}$ is isomorphic to $S O(3)$ and the action of $L_{\theta}$ on $\boldsymbol{C} \boldsymbol{a}_{0}$ is equivalent to the direct sum of the adjoint action of $S O(3)$ on $\mathfrak{g n}(3, \boldsymbol{C})$ and the trivial action of $S O(3)$ on $\boldsymbol{R}$. Therefore by calculating the volume of each orbit $([6])$, we can easily see that the only orbit through $p=\left(2^{1 / 2} / 2\right)\left(e_{2}+e_{5}\right)$ is a 3 -dimensional minimal submanifold of $S^{6}$ under the action of $L_{\theta}$ on $\boldsymbol{C a}_{0}$. The tangent space of the orbit at $p$ is spanned by

$$
\begin{aligned}
& X_{1}(p)=2^{1 / 2}\left(e_{1}-e_{6}\right) \\
& X_{2}(p)=-2^{1 / 2}(\cos \theta) e_{3}-2^{1 / 2}(\sin \theta) e_{7}, \\
& X_{3}(p)=2^{1 / 2}(\sin \theta) e_{3}-2^{1 / 2}(\cos \theta) e_{7} .
\end{aligned}
$$

Consulting the multiplication table (1.1), we get

$$
\begin{aligned}
& J\left(X_{1}(p)\right)=p \cdot X_{1}(p)=2 e_{4}, \\
& J\left(X_{2}(p)\right)=p \cdot X_{2}(p)=-\cos \theta\left(e_{1}+e_{6}\right)+\sin \theta\left(-e_{2}+e_{5}\right),
\end{aligned}
$$




$$
J\left(X_{3}(p)\right)=p \cdot X_{3}(p)=\cos \theta\left(-e_{2}+e_{5}\right)+\sin \theta\left(e_{1}+e_{6}\right) .
$$

Therefore the orbit is a totally real submanifold.

Q.E.D.

CASE IV. For this case we have the following

THEOREM 4.3. Let $L$ be the subgroup of $G_{2}$ generated by the Lie subalgebra spanned by $X_{1}, X_{2}$ and $X_{3}$ defined by (3.8). Then, under the action of $L$ on $\boldsymbol{C a}_{0}$, there exist exactly 2-types of orbits in $S^{6}$ which are 3-dimensional totally real submanifold of $S^{6}$ up to the action of $G_{2}$. They are

(1) the orbit through $e_{2}$, which we denote by $M_{3}$.

(2) the orbit through $e_{6}$, which we denote by $M_{4}$.

It is easily seen that $M_{3}$ is of constant curvature $1 / 16$. The proof of this Theorem will be given in $\S 6$.

Let $M$ be a compact 3-dimensional totally real submanifold of $S^{6}$, which is obtained as an orbit of a closed subgroup $L$ of $G_{2}$. It is well-known that the dimension of $L$ is smaller than or equal to 6 ([10]). If $\operatorname{dim} L=6$, then $M$ is a space of constant curvature and, by a Theorem of Ejiri, the curvature of $M$ is $1 / 16([3])$. And by Theorem 2.5 it (if exists) is congruent to $M_{3}$ of Theorem 4.3. It is known that if $\operatorname{dim} L \leqq 5$, then $\operatorname{dim} L \leqq 4([10])$. If $\operatorname{dim} L=4$, then the Lie algebra $\mathfrak{l}$ of $L$ must be isomorphic to $\mathfrak{u}(2)$, since $L$ is compact. By a direct calculation we see that it is isomorphic to the Lie subalgebra of $\mathbb{B}_{2}$ which is spanned by

$$
\begin{aligned}
& X_{1}=-2 G_{23}+G_{45}+G_{76}, \\
& X_{2}=-2 G_{31}+G_{46}+G_{57}, \\
& X_{3}=-2 G_{12}+G_{47}+G_{65}, \\
& J=a\left(G_{45}-G_{76}\right)+b\left(G_{46}-G_{57}\right)+b\left(G_{47}-G_{65}\right), a, b, c \in \boldsymbol{R} .
\end{aligned}
$$

Let $G_{3}$ be the Lie subgroup of $L$ whose Lie algebra is $\boldsymbol{R} X_{1}+\boldsymbol{R} X_{2}+\boldsymbol{R} X_{3}$. Then it is easily seen that $L(p)=G_{s}(p)$ for any $p \in S_{1}^{6}$. Thus we have the following

TheOREM 4.4. Let $M$ be a 3-dimensional totally real submanifold of $S_{1}^{6}$ which is obtained as an orbit of a closed subgroup of $G_{2}$. Then $M$ is congruent to one of the $M_{1}, M_{2}, M_{3}$ or $M_{4}$, unless it is a great sphere.

\section{$\S 5$. Orbits in a sphere}

In this section we prepare some Lemmata to prove Theorem 4.3,

Let $G$ be a Lie subgroup of $S O(N+1)$. Then $G$ acts on the unit sphere $S_{1}^{N}$ in $\boldsymbol{R}^{N+1}$ centered at the origin in a natural manner. Take a point $p$ in $S_{1}^{N}$ and let 
$M$ be the orbit of the action of $G$ through $p$.

Let $\&$ be the Lie algebra of $G$. We denote by $A^{*}$ the vector field on $S_{1}^{N}$ induced by $A \in \mathbb{S}$. Then, by regarding $A$ as a skew symmetric transfomation on $\boldsymbol{R}^{N+1}$, we have

$$
A^{*}{ }_{\mid p}=A(p), \quad A \in \mathbb{B}, \quad p \in S_{1}^{N} .
$$

Therefore the tangent space of $M$ at $p$ is

$$
T_{p}(M)=\{A(p) \mid A \in \mathbb{\&}\} .
$$

Let $N_{p}(M)$ be the normal space of $M$ in $S_{1}^{N}$ at $p$. Regard the tangent space $T_{p}(M)$ and the normal space $N_{p}(M)$ as subspaces of $\boldsymbol{R}^{N+1}$. Then $\boldsymbol{R}^{N+1}$ is decomposed into the direct sum

$$
\boldsymbol{R}^{N+1}=\boldsymbol{R} p+T_{p}(M)+N_{p}(M) .
$$

For a vector $X$ in $\boldsymbol{R}^{N+1}$, we denote by $X^{T}\left(\operatorname{resp} . X^{N}\right)$ the $T_{p}(M)-\left(\operatorname{resp} . N_{p}(M)-\right)$ component of $X$ with respect to the decomposition (5.1).

LemMA 5.1. Let $G$ be a Lie subgroup of $S O(N+1)$. Let $\alpha$ be the second fundamental form of the orbit $M=G(p)$. Then

$$
\begin{aligned}
& \alpha\left(A^{*}, B^{*}\right)_{\mid p}=(A(B(p)))^{N}, \\
& \nabla_{B^{*}} A^{*}{ }_{\mid p}=(A(B(p)))^{T}, \quad A, B \in \mathbb{B} .
\end{aligned}
$$

where $\nabla$ is the Riemannian connection of $M$.

Proof. Let $D$ be the Riemannian connection of $\boldsymbol{R}^{N+1}$. Then

$$
\begin{aligned}
D_{B^{*}} A^{*}{ }_{1 p} & =d / d t_{\mid t=0} A^{*}{ }_{\mid \exp (t B)(p)} \\
& =d / d t_{\mid t=0} A(\exp t B)(p) \\
& =A(B(p)) .
\end{aligned}
$$

Since $\alpha\left(A^{*}, B^{*}\right)_{\mid p}=\left(D_{B^{*}} A^{*}{ }_{\mid p}\right)^{N}$ and $\nabla_{B^{*}} A^{*}{ }_{\mid p}=\left(D_{B^{*}} A^{*}{ }_{\mid p}\right)^{T}$, we get $(5.2)$ and $(5.3)$,

Q.E.D.

LEMMA 5.2. Let $G$ be a Lie subgroup of $S O(N+1)$ and fix an orbit $M=G(p)$. Let $S$ be the complete connected totally geodesic submanifold of $S_{1}^{N}$ such that $T_{p}(S)$ $=N_{p}(M)$. Then each $G$-orbit in $S_{1}^{N}$ contains at least one point of $S$.

Proof. Take an arbitrary orbit $M^{\prime}=G\left(p^{\prime}\right)$. Then there exists a point $p_{1}$ in $M$ and a point $p_{2}$ in $M^{\prime}$ such that the distance between $M$ and $M^{\prime}$ is attained by $p_{1}$ and $p_{2}$. Let $\tau$ be the shortest geodesic joining $p_{1}$ and $p_{2}$. Take an element $\sigma \in G$ such that $\sigma\left(p_{1}\right)=p$. Since $\sigma$ is an isometry of $S_{1}^{N}, \sigma(\tau)$ is also a geodesic and 
is normal to $M$ at $p$. Therefore $\sigma(\tau)$ is contained in $S$ and $\sigma\left(p_{2}\right)$ is contained in $S \cap M$.

Q.E.D.

Now we consider the case that $G$ is isomorphic to $S U(2)$ or $S O(3)$. Let $B$ be the Killing form of $\mathfrak{g} \mathfrak{u}(2)$. Then the basis $X_{1}, X_{2}$ and $X_{3}$ with $\left[X_{1}, X_{2}\right]=2 X_{3}$, $\left[X_{2}\right.$, $\left.X_{3}\right]=2 X_{1}$ and $\left[X_{3}, X_{1}\right]=2 X_{2}$ is orthonormal with respect to $-B / 8$. Let $g_{0}$ be the Riamannian metric on $G$ which is the bi-invariant extension of $-B / 8$.

LeMma 5.3 (Sugahara, [9]). Let $g$ be an inner product on $\mathfrak{g} u(2)$. Then there exists an element $\sigma$ in $G$ such that

(i ) $X_{i}^{\prime}=A d(\sigma)\left(X_{i}\right), i=1,2,3$, are mutually orthogonal with respect to $g$.

(ii) $g=\lambda_{1} \omega_{1}{ }^{2}+\lambda_{2} \omega_{2}{ }^{2}+\lambda_{3} \omega_{3}{ }^{2}$, where $\lambda_{i}$ are positive constants and $\omega_{i}(\cdot)=g_{0}\left(X_{i}, \cdot\right), i=$ $1,2,3$.

Remark 5.4. (i ) Put $\sigma=\exp \left(\pi X_{i} / 4\right)$. Then $\operatorname{Ad}(\sigma)\left(X_{1}\right)=X_{1}, \quad \operatorname{Ad}(\sigma)\left(X_{2}\right)=X_{3}$ and $A d(\sigma)\left(X_{3}\right)=-X_{2}$ so that $\lambda_{2}$ and $\lambda_{3}$ of Lemma 5.3 can be permuted. Similarly $\lambda_{1}$ and $\lambda_{2}$ (resp. $\lambda_{1}$ and $\left.\lambda_{3}\right)$ are permuted by $\operatorname{Ad}\left(\exp \left(\pi X_{3} / 4\right)\right)$ (resp. $\left.A d\left(\exp \left(\pi X_{2} / 4\right)\right)\right)$.

(ii) $(G, g)$ is a space of constant curvature $k$ if and only if $\lambda_{1}=\lambda_{2}=\lambda_{3}=1 / k$, i.e., $g=(1 / k) g_{0}$.

Lemma 5.5 (Sugahara, [9]). Let $X_{1}^{\prime}, X_{2}^{\prime}$ and $X_{3}^{\prime}$ be as in Lemma 5.3. Then the one parameter subgroups $\tau_{x_{i}}(t)=\exp t X_{i}, i=1,2,3$, are geodesics of $(G, g)$.

Let $(V, \rho)$ be an orthogonal representation of $G$ and $\langle$,$\rangle be a G$-invariant inner product on $V$. Let $M=G(p)$ be an orbit in the unit sphere $S_{1}$ through $p$.

Lemma 5.6. If $\operatorname{dim} M=3$, then there exists an element $\sigma$ in $G$ such that

$$
\left\langle\rho\left(X_{i}\right)(\sigma(p)), \rho\left(X_{j}\right)(\sigma(p))\right\rangle=0 \text { for } i \neq j .
$$

Proof. Define a map $f: G \longrightarrow S_{1}$ by

$$
f(\sigma)=\rho(\sigma)(p), \quad \sigma \in G .
$$

Then $f_{*}\left(X_{i}\right)=\rho\left(X_{i}\right)(p)$. Let $g$ be the metric on $G$ induced by $f$. Then $g$ is a left invariant metric. Consider the inner product $g_{l e}$ on the tangent space $T_{e}(G)$ at the unit element $e$. Then by Lemma 5.3 there exists an element $\sigma$ in $G$ such that $A d\left(\sigma^{-1}\right)\left(X_{i}\right), i=1,2,3$, are mutually orthogonal with respect $g_{1 e}$. Let $R_{\sigma}$ and $L_{\sigma}$ be the right and left translations by $\sigma$ respectively. Then we have

$$
f_{*}\left(d R_{\sigma}(X)\right)=d / d t_{\mid t=0} f(\exp (t X) \sigma(p))=\rho(X)(\sigma(p)), X \in \mathfrak{g} \mathfrak{u}(2), p \in S_{1} .
$$

Since $A d\left(\sigma^{-1}\right)\left(X_{i}\right)$ and $A d\left(\sigma^{-1}\right)\left(X_{j}\right)$ are orthogonal if $i \neq j$, it follows from (5.5) that

$$
0=g\left(A d\left(\sigma^{-1}\right)\left(X_{i}\right), \quad A d\left(\sigma^{-1}\right)\left(X_{j}\right)\right)
$$




$$
\begin{aligned}
& =g\left(d L_{\sigma^{-1}}\left(d R_{\sigma}\left(X_{i}\right)\right), \quad d L_{\sigma^{-1}}\left(d R_{\sigma}\left(X_{j}\right)\right)\right) \\
& \left.=g\left(d R_{\sigma}\left(X_{i}\right)\right), \quad d R_{\sigma}\left(X_{j}\right)\right) \\
& =\left\langle f_{*}\left(d R_{\sigma}\left(X_{i}\right)\right), \quad f_{*}\left(d R_{\sigma}\left(X_{j}\right)\right)\right\rangle \\
& =\left\langle\rho\left(X_{i}\right)(\sigma(p)), \quad \rho\left(X_{j}\right)(\sigma(p))\right\rangle .
\end{aligned}
$$

Hereafter we may assume

$$
\left\langle\rho\left(X_{i}\right)(p), \rho\left(X_{j}\right)(p)\right\rangle=0, i \neq j,
$$

if the orbit $M=G(p)$ is of dimension 3 .

Lemma 5.7. Let $M=G(p)$ be a 3-dimensional orbit. Then $f: G \longrightarrow S_{1}$ defined by (5.4) is a minimal immersion if and only if

$$
\sum_{i=1}^{3} X_{i}\left(X_{i}(p)\right) / \lambda_{i}=-3 p,
$$

where $\lambda_{i}=\left\langle X_{i}(p), X_{i}(p)\right\rangle, i=1,2,3$.

Proof. Since (5.6) holds at the initial point $p, X_{i}^{\prime}=X_{i} / \lambda_{i}{ }^{1 / 2}$ is an orthonormal frame of $T_{e}(G)$. By the $G$-equivalence of the immersion $f$, we have only to verify $\sum_{i=1}^{3} \alpha\left(X_{i}^{\prime}, X_{i}^{\prime}\right)_{\mid e}=0$. Since $\tau_{X_{i}^{\prime}}(t)=\exp t X_{i}^{\prime}$ are geodesics of $(G, g)$, by (5.2) we get

$$
\nabla_{X_{i}} * X_{i}{ }^{*}{ }_{1 e}=\left(X_{i}\left(X_{i}(p)\right)^{T}=0 .\right.
$$

By (5.3) $f$ is a minimal immersion if and only if $\sum_{i=1}^{3} \alpha\left(X_{i}^{\prime}, X_{i}^{\prime}\right)=0$. Therefore $\sum_{i=1}^{3} X_{i}^{\prime}\left(X_{i}^{\prime}(p)\right)$ is proportional to $p$ if and only if $f$ is a minimal immersion.

Now we assume that $\sum_{i=1}^{3} X_{i}^{\prime}\left(X_{i}^{\prime}(p)\right)=\mathrm{cp}$ for some constant $c$. Then

$$
\begin{aligned}
c & =\left\langle\sum_{i=1}^{3} X_{i}\left(X_{i}(p)\right) / \lambda_{i}, p\right\rangle \\
& =-\sum_{i=1}^{3}\left\langle X_{i}(p), X_{i}(p)\right\rangle / \lambda_{i} \\
& =-3 .
\end{aligned}
$$

\section{§6. Proof of Theorem 4.3}

Let $L$ be the Lie subgroup of $G_{2}$ generated by the Lie subalgebra defined by (3.8) and let $p=\sum_{j=1}^{7} x_{j} e_{j}$ be a point on $S_{1}^{6}$. Then the tangent space of $L(p)$ is spanned by

$$
\begin{aligned}
X_{1}(p)= & -4 x_{3} e_{2}+4 x_{2} e_{3}-2 x_{5} e_{4}+2 x_{4} e_{5}-6 x_{7} e_{6}+6 x_{6} e_{7}, \\
X_{2}(p)= & -2 \cdot 6^{1 / 2} x_{5} e_{1}+\left(6^{1 / 2} x_{6}-10^{1 / 2} x_{4}\right) e_{2}+\left(6^{1 / 2} x_{7}-10^{1 / 2} x_{5}\right) e_{3} \\
& +10^{1 / 2} x_{2} e_{4}+\left(2 \cdot 6^{1 / 2} x_{1}+10^{1 / 2} x_{3}\right) e_{5}-6^{1 / 2} x_{2} e_{6}-6^{1 / 2} x_{3} e_{7},
\end{aligned}
$$




$$
\begin{aligned}
X_{3}(p)= & 2 \cdot 6^{1 / 2} x_{4} e_{1}+\left(6^{1 / 2} x_{7}+10^{1 / 2} x_{5}\right) e_{2}-\left(6^{1 / 2} x_{6}+10^{1 / 2} x_{4}\right) e_{3} \\
& +\left(10^{1 / 2} x_{3}-2 \cdot 6^{1 / 2} x_{1}\right) e_{4}-10^{1 / 2} x_{2} e_{5}+6^{1 / 2} x_{3} e_{6}-6^{1 / 2} x_{2} e_{7}
\end{aligned}
$$

We may assume that (5.6) holds at $p$, i.e.,

$$
\begin{aligned}
5\left(x_{3} x_{6}-x_{2} x_{7}\right)+15^{1 / 2}\left(x_{2} x_{5}-x_{3} x_{4}\right)-2 x_{1} x_{4}=0, \\
5\left(x_{2} x_{6}+x_{3} x_{7}\right)+15^{1 / 2}\left(x_{2} x_{4}+x_{3} x_{5}\right)-2 x_{1} x_{5}=0, \\
15^{1 / 2}\left(2 x_{1} x_{2}+x_{4} x_{7}-x_{5} x_{6}\right)+6 x_{4} x_{5}=0 .
\end{aligned}
$$

Then by Lemma 5.5, the orbit $L(p)$ is a minimal submanifold of $S_{1}^{6}$ if and only if

$$
\begin{array}{ll}
-24\left(1 / \lambda_{2}+1 / \lambda_{3}\right) x_{1}-4 \cdot 15^{1 / 2}\left(1 / \lambda_{2}-1 / \lambda_{3}\right) x_{3} & =-3 x_{1}, \\
-16\left(1 / \lambda_{1}+1 / \lambda_{2}+1 / \lambda_{3}\right) x_{2} & =-3 x_{2}, \\
-16\left(1 / \lambda_{1}+1 / \lambda_{2}+1 / \lambda_{3}\right) x_{3}-4 \cdot 15^{1 / 2}\left(1 / \lambda_{2}-1 / \lambda_{3}\right) x_{1}=-3 x_{3}, & \\
-\left(4 / \lambda_{1}+10 / \lambda_{2}+34 / \lambda_{3}\right) x_{4}+2 \cdot 15^{1 / 5}\left(1 / \lambda_{2}-1 / \lambda_{3}\right) x_{7}=-3 x_{4}, & \\
-\left(4 / \lambda_{1}+34 / \lambda_{2}+10 / \lambda_{3}\right) x_{5}+2 \cdot 15^{1 / 2}\left(1 / \lambda_{2}-1 / \lambda_{3}\right) x_{7}=-3 x_{5}, & =-3 x_{6}, \\
-\left(36 / \lambda_{1}+6 / \lambda_{2}+6 / \lambda_{3}\right) x_{6}+2 \cdot 15^{1 / 2}\left(1 / \lambda_{2}-1 / \lambda_{3}\right) x_{4} & =-3 x_{7}, \\
-\left(36 / \lambda_{1}+6 / \lambda_{2}+6 / \lambda_{3}\right) x_{7}+2 \cdot 15^{1 / 2}\left(1 / \lambda_{2}-1 / \lambda_{3}\right) x_{5} & =-3 x^{2}
\end{array}
$$

where

$$
\begin{aligned}
\lambda_{1} & =16\left(x_{2}{ }^{2}+x_{3}{ }^{2}\right)+4\left({x_{4}}^{2}+x_{5}{ }^{2}\right)+36\left(x_{6}{ }^{2}+x_{7}{ }^{2}\right), \\
\lambda_{2} & =24 x_{1}{ }^{2}+16\left(x_{2}{ }^{2}+x_{3}{ }^{2}\right)+10 x_{4}{ }^{2}+34 x_{5}{ }^{2}+6\left(x_{6}{ }^{2}+x_{7}{ }^{2}\right) \\
& +4 \cdot 15^{1 / 2}\left(2 x_{1} x_{3}-x_{4} x_{6}-x_{5} x_{7}\right), \\
\lambda_{3} & =24 x_{1}{ }^{2}+16\left(x_{2}{ }^{2}+x_{3}{ }^{2}\right)+34 x_{4}{ }^{2}+10 x_{5}{ }^{2}+6\left(x_{6}{ }^{2}+x_{7}{ }^{2}\right) \\
& -4 \cdot 15^{1 / 2}\left(2 x_{1} x_{3}-x_{4} x_{6}-x_{5} x_{7}\right) .
\end{aligned}
$$

LeMmA 6.1. If $x_{1}, x_{2}, \cdots, x_{7}, \sum_{i=1}^{7} x_{i}{ }^{2}=1$, satisfy (6.1) and (6.2) then $\left(\lambda_{1}, \lambda_{2}, \lambda_{3}\right)$ is $(16,16,16),(36,6,6)$ or $\left(20+4 \cdot 15^{1 / 2}, 8,20-4 \cdot 15^{1 / 2}\right)$ up to permutation.

Proof. By adding $(6.3)_{1},(6.3)_{2}$, and $(6.3)_{3}$, we get

$$
\lambda_{1}+\lambda_{2}+\lambda_{3}=48\left(x_{1}{ }^{2}+\cdots+x_{7}^{2}\right)=48 \text {. }
$$

If $x_{2} \neq 0$, then we get $1 / \lambda_{1}+1 / \lambda_{2}+1 / \lambda_{3}=3 / 16$ from $(6.2)_{2}$. Thus we have

$$
\begin{aligned}
16 & =\left(\lambda_{1}+\lambda_{2}+\lambda_{3}\right) / 16 \geqq\left(\lambda_{1} \lambda_{2} \lambda_{3}\right)^{1 / 2} \\
& \geqq 3 /\left(1 / \lambda_{1}+1 / \lambda_{2}+1 / \lambda_{3}\right)=16 .
\end{aligned}
$$


The equalities hold if and only if $\lambda_{1}=\lambda_{2}=\lambda_{3}=16$. Hereafter we assume $x_{2}=0$.

CASE $1 . \quad \lambda_{i}=\lambda_{j}$ for some $i, j, 1 \leqq i \neq j \leqq 3$.

By Remark 5.2, we may assume $\lambda_{2}=\lambda_{3}$ without loss of generality.

If $x_{1} \neq 0$, then we get $\lambda_{1}=\lambda_{2}=\lambda_{3}=16$ by $(6.2)_{1}$, and (6.4). If $x_{3} \neq 0$, we get $\lambda_{1}=$ $\lambda_{2}=\lambda_{3}=16$ by an argument similar to the case of $x_{2} \neq 0$. If $x_{4} \neq 0$ or $x_{5} \neq 0$, we get $\left(\lambda_{1}, \lambda_{2}, \lambda_{3}\right)=(16,16,16)$ or $(4,22,22)$. If $\left(\lambda_{1}, \lambda_{2}, \lambda_{3}\right)=(4,22,22)$, then $x_{1}=x_{2}=x_{3}=x_{6}$ $=x_{7}=0$ by $(6.2)_{1},(6.2)_{2},(6.2)_{3},(6.2)_{6}$ and $(6.2)_{7}$ so that $x_{4} \cdot x_{5}=0$ by $(6.1)_{3}$. But from $(6.3)_{2}$ and $(6.3)_{3}$, we get $x_{4}{ }^{2}=x_{5}{ }^{2}$. This is a contradiction. Thus we have $\left(\lambda_{1}, \lambda_{2}, \lambda_{3}\right) \neq(4,22,22)$. If $x_{6} \neq 0$ or $x_{7} \neq 0$, then we get $\left(\lambda_{1}, \lambda_{2}, \lambda_{3}\right)=(16,16,16)$ or $(36,6,6)$.

CASE 2. $\lambda_{1}, \lambda_{2}$ and $\lambda_{3}$ are mutually different.

In this case we may assume $\lambda_{1}>\lambda_{2}>\lambda_{3}$ by Remark 5.2.

If $x_{1}=0$ (resp. $x_{3}=0$ ), then $x_{3}=0$ by $(6.2)_{1}$ (resp. $x_{1}=0$ by $(6.2)_{3}$ ).

If $x_{4}=0$ (resp. $x_{6}=0$ ), then $x_{6}=0$ by $(6.2)_{4}$ (resp. $x_{4}=0$ by $(6.2)_{6}$ ).

If $x_{5}=0$ (resp. $x_{7}=0$ ), then $x_{7}=0$ by $(6.2)_{5}$ (resp. $x_{5}=0$ by $(6.2)_{7}$ ).

By $(6.2)_{6}$ and $(6.2)_{7}$, we get

$$
x_{4} x_{7}-x_{5} x_{6}=0 \text {. }
$$

By $(6.2)_{4}$ and $(6.2)_{5}$, we get

$$
2 \cdot 15^{1 / 2}\left(1 / \lambda_{2}-1 / \lambda_{3}\right)\left(x_{5} x_{6}-x_{4} x_{7}\right)-24\left(1 / \lambda_{3}-1 / \lambda_{2}\right) x_{4} x_{5}=0 .
$$

Thus we have $x_{4} x_{5}=0$. Finally we have the following five subcases.

Subcase 2.1. $x_{2}=x_{5}=x_{7}=0, x_{1} x_{3} x_{4} x_{6} \neq 0$.

Subcase 2.2. $x_{2}=x_{4}=x_{5}=x_{6}=x_{7}=0, x_{1} x_{3} \neq 0$.

Subcase 2.3. $x_{1}=x_{2}=x_{3}=x_{4}=x_{6}=0, x_{5} x_{7} \neq 0$.

Subcase 2.4. $x_{2}=x_{4}=x_{6}=0, x_{1} x_{3} x_{5} x_{7} \neq 0$.

Subcase 2.5. $x_{1}=x_{2}=x_{3}=x_{5}=x_{7}=0, x_{4} x_{6} \neq 0$.

SubCASE 2.1. Put $\mu_{i}=1 / \lambda_{i}, i=1,2,3$. Since (6.2) $)_{1}$ and (6.2) ${ }_{3}$ (resp. (6.2) ${ }_{4}$ and $\left.(6.2)_{s}\right)$ have a non-trivial solution $\left(x_{1}, x_{3}\right)$ (resp. $\left(x_{4}, x_{6}\right)$ ), we get

$$
\begin{aligned}
0 & =(1 / 3) \operatorname{det}\left[\begin{array}{ll}
3-24\left(\mu_{2}+\mu_{3}\right) & 4 \cdot 15^{1 / 2}\left(\mu_{2}-\mu_{3}\right) \\
4 \cdot 15^{1 / 2}\left(\mu_{2}-\mu_{3}\right) & 3-16\left(\mu_{1}+\mu_{2}+\mu_{3}\right)
\end{array}\right] \\
& =3-8\left(2 \mu_{1}+5 \mu_{2}+5 \mu_{3}\right)+128\left(\mu_{2}+\mu_{3}\right)\left(\mu_{1}+\mu_{2}+\mu_{3}\right)-80\left(\mu_{2}-\mu_{3}\right)^{2}, \\
0 & =(1 / 3) \operatorname{det}\left[\begin{array}{ll}
3-\left(4 \mu_{1}+10 \mu_{2}+34 \mu_{3}\right) & 2 \cdot 15^{1 / 2}\left(\mu_{2}-\mu_{3}\right) \\
2 \cdot 15^{1 / 2}\left(\mu_{2}-\mu_{3}\right) & 3-\left(36 \mu_{1}+6 \mu_{2}+6 \mu_{3}\right)
\end{array}\right]
\end{aligned}
$$




$$
=3-8\left(5 \mu_{1}+2 \mu_{2}+5 \mu_{3}\right)+4\left(2 \mu_{1}+17 \mu_{2}+5 \mu_{3}\right)\left(6 \mu_{1}+\mu_{2}+\mu_{3}\right)-20\left(\mu_{2}-\mu_{3}\right)^{2} .
$$

By subtracting $(6.5)_{2}$ from $(6.5)_{1}$, we get

$$
0=\left(\mu_{1}-\mu_{2}\right)\left(1-2 \mu_{1}-2 \mu_{2}-12 \mu_{3}\right) \text {. }
$$

Since $\mu_{1}>\mu_{2}$, we get

$$
\mu_{1}+\mu_{2}=1 / 2-6 \mu_{3} .
$$

By adding $(6.5)_{1}$ and $(6.5)_{2}$, we get

$$
0=3-28\left(\mu_{1}+\mu_{2}\right)-40 \mu_{3}+24\left(\mu_{1}+\mu_{2}\right)^{2}+48 \mu_{3}^{2}+80 \mu_{1} \mu_{2}+272\left(\mu_{1}+\mu_{2}\right) \mu_{3} .
$$

By substituting (6.6) into (6.7), we get

$$
0=-\left(1-12 \mu_{3}\right)^{2}+16 \mu_{1} \mu_{2} .
$$

From (6.4) and (6.6), we obtain

$$
\mu_{1} \mu_{2}=\mu_{3}\left(1-12 \mu_{3}\right) / 2\left(48 \mu_{3}-1\right) .
$$

Therefore we get

$$
0=-\left(1-12 \mu_{3}\right)^{2}+8 \mu_{3}\left(1-12 \mu_{3}\right) /\left(48 \mu_{3}-1\right) .
$$

As solutions of the above equation, we get $\lambda_{3}=1 / \mu_{3}=12,16,36$. If $\mu_{3}=1 / 12$, we get $\mu_{1}=\mu_{2}=0$ from $(6.6)$, Thus $\lambda_{3} \neq 12$. For $\lambda_{3}=16,36$ we get $\left(\lambda_{1}, \lambda_{2}, \lambda_{3}\right)=(16,16$, 16), $(36,6,6)$ by $(6.6)$ and (6.8) respectively. Therefore Subcase 2.1 cannot occur.

SubCASE 2.2. By $(6.2)_{1},(6.3)_{2}$ and $(6.3)_{3}$, we get

$$
16 x_{1}{ }^{4}-18{x_{1}}^{2}+5=0 \text {. }
$$

As solutions of the above equation we get $x_{1}^{2}=5 / 8,1 / 2$. If $x_{1}{ }^{2}=5 / 8$, then $\left(\lambda_{1}, \lambda_{2}\right.$, $\left.\lambda_{3}\right)=(6,36,6),(6,6,36)$. Thus $x_{1}{ }^{2} \neq 5 / 8$. If $x_{1}{ }^{2}=1 / 2,\left(\lambda_{1}, \lambda_{2}, \lambda_{3}\right)=\left(8,20+4 \cdot 15^{1 / 2}\right.$, $\left.20-4 \cdot 15^{1 / 2}\right)$.

SUBCASE 2.3. Let $p=x_{5} e_{5}+x_{7} e_{7}$ be a solution of (6.1) and (6.2). Then from Remark 5.2, $\exp \left(\pi X_{2} / 4\right)(p)$ is also a solution and $\lambda^{\prime} s$ for $\exp \left(\pi X_{2} / 4\right)(p)$ coincide (up to permutation) with $\lambda$ 's for $p$.

It is easily seen that $V_{1}=\boldsymbol{R} e_{1}+\boldsymbol{R} e_{3}+\boldsymbol{R} e_{5}+\boldsymbol{R} e_{7}$ is invariant under $\exp \left(\pi X_{3} / 4\right)$. We can see that the restriction $\exp \left(\pi X_{3} / 4\right) \mid V_{1}$ is $\left[\begin{array}{ccc}* & & * \\ * & 0 & 0 \\ & 0 & 0\end{array}\right]$ with respect to $e_{1}, e_{3}$, $e_{5}, e_{7}$. Thus Subcase 2.3 is reduced to the Subcase 2.2.

SubCASE 2.4. Let $\left(x_{1}, 0, x_{3}, 0, x_{5}, 0, x_{7}\right), x_{1} x_{3} x_{5} x_{7} \neq 0$, be a solution of (6.1) and $(6.2)$ with $\left(\lambda_{1}, \lambda_{2}, \lambda_{3}\right)=(a, b, c)$. Then $\left(-x_{1}, 0, x_{3},-x_{5}, 0, x_{7}, 0\right)$ is also a solu- 
tion of (6.1) and (6.2) with $\left(\lambda_{1}, \lambda_{2}, \lambda_{3}\right)=(a, c, b)$. Thus Subcase 2.4 is reduced to Subcase 2.1 .

Subcase 2.5. By an argument similar to Subcase 2.3, Subcase 2.5 is reduced to Subcase 2.2 .

Q.E.D.

Now we prove the existence and uniqueness (up to the action of $G_{2}$ ) of orbits in $S_{1}^{6}$ which are minimal submanifolds of $S_{1}^{6}$ and $\left(\lambda_{1}, \lambda_{2}, \lambda_{3}\right)$ of Lemma 6.1 is (16, $16,16),(36,6,6)$ or $\left(4,20-4 \cdot 15^{1 / 2}, 20+4 \cdot 15^{1 / 2}\right)$. First we prove the following

LEMMA 6.2. There exists an orbit which is a totally real submanifold of $S_{1}^{6}$ and $\left(\lambda_{1}, \lambda_{2}, \lambda_{3}\right)$ of Lemma 6.1 is $(16,16,16)$. Such an orbit is unique up to the action of $G_{2}$.

Proof. Put $\left(x_{1}, x_{2}, \cdots, x_{7}\right)=(0,1,0, \cdots, 0)$. Then we can easily verify that $\left(x_{1}, \cdots, x_{7}\right)$ is a solution of $(6.1)$ and (6.2) with $\lambda_{1}=\lambda_{2}=\lambda_{3}=16$.

Apply the Lemma 5.2 to the orbit $M_{4}=L\left(e_{6}\right)$. Then each orbit contains at least one point of $S=\left\{x_{1} e_{1}+x_{4} e_{4}+x_{5} e_{5}+x_{6} e_{6} \mid x_{1}{ }^{2}+x_{4}{ }^{2}+x_{5}{ }^{2}+x_{6}{ }^{2}=1\right\}$.

Assume that an orbit $M$ through $p=x_{1} e_{1}+x_{4} e_{4}+x_{5} e_{5}+x_{6} e_{6}$ is a minimal submanifold of $S_{1}^{6}$ with $\lambda_{1}=\lambda_{2}=\lambda_{3}=16$. Then, since the induced metric on $L$ is bi-invariant, (5.6) must hold at any point on the orbit. Therefore we get

$$
x_{1} x_{4}=x_{1} x_{5}=-15^{1 / 2} x_{5} x_{6}+6 x_{4} x_{5}=0 .
$$

Under the above conditions, we solve the equation $\lambda_{1}=\lambda_{2}=\lambda_{3}=16$. Then we have $\left(x_{1}, x_{4}, x_{5}, x_{6}\right)= \pm\left(0,10^{1 / 2} / 4,0, \pm 6^{1 / 2} / 2\right), \pm\left(5^{1 / 2} / 3,0,0, \pm 2 / 3\right)$ or $\pm\left(0,10^{1 / 2} / 4\right.$, $\left.\pm 6^{1 / 2} / 4, \pm 30^{1 / 2} / 8\right)$. It is easily verified that an orbit through each of the above points is a totally real submanifold of $S_{1}^{6}$ and of constant curvature $1 / 16$. Thus by Lemma 2.5, they are congruent under the action of $G_{2}$.

Q.E.D.

LEMMA 6.3. There exists a unique orbit which is a totally real submanifold of $S_{1}^{6}$ and $\left(\lambda_{1}, \lambda_{2}, \lambda_{3}\right)$ of Lemma 6.1 is $(36,6,6)$ up to permutation.

Proof. By Remark 5.2, we may assume that $\lambda_{1}=36, \lambda_{2}=\lambda_{3}=6$. Since $\lambda_{1}=36$, (6.3), and (6.4) yield

$$
\begin{aligned}
0 & =36\left(x_{1}{ }^{2}+\cdots+x_{7}{ }^{2}\right)-16\left({x_{2}}^{2}+x_{3}{ }^{2}\right)-4\left(x_{4}{ }^{2}+x_{5}{ }^{2}\right)-36\left(x_{6}{ }^{2}+x_{7}{ }^{2}\right) \\
& =36 x_{1}{ }^{2}+20\left(x_{2}{ }^{2}+x_{3}{ }^{2}\right)+32\left(x_{4}{ }^{2}+x_{5}{ }^{2}\right)
\end{aligned}
$$

so that $x_{i}=0, i=1, \cdots, 5$. It is easily verified that $\left(x_{1}, \cdots, x_{5}, x_{6}, x_{7}\right)=(0, \cdots, 0$, $\cos \theta, \sin \theta)$ is a solution of $(6.1)$ and $(6.2)$ with $\lambda_{1}=36, \lambda_{2}=\lambda_{3}=6$. By a simple computation, we get $\exp \left(\theta X_{3} / 6\right)\left(e_{6}\right)=(\cos \theta) e_{6}+(\sin \theta) e_{7}$. Hence these points are contained in exactly one orbit. Furthermore we can easily see that this orbit is 
a totally real submanifold of $S_{1}^{6}$.

Q.E.D.

Lemma 6.5. There exists a minimal submanifold of $S_{1}^{6}$ such that $\left(\lambda_{1}, \lambda_{2}, \lambda_{3}\right)$ of Lemma 6.1 is equal to $\left(8,20+4 \cdot 15^{1 / 2}, 20-4 \cdot 15^{1 / 2}\right)$. But it is not a totally real submanifold.

PROof. It is easily verified that an orbit through each of the points $\pm\left(2^{1 / 2} / 2\right.$, $\left.0, \pm 2^{1 / 2} / 2,0, \cdots, 0\right)$ is a minimal submanifold of $S_{1}^{6}$. In the way of proving Lemma 6.1 , we proved that any orbit in $S_{1}^{6}$ which is a minimal submanifold such that $\left(\lambda_{1}\right.$, $\left.\lambda_{2}, \lambda_{3}\right)$ is equal to $\left(8,20+4 \cdot 15^{1 / 2}, 20-4 \cdot 15^{1 / 2}\right)$ is congruent to one of the orbits through $\pm\left(2^{1 / 2} / 2,0, \pm 2^{1 / 2} / 2,0, \cdots, 0\right)$ under the action of $G_{2}$. But by direct calculations, they are not totally real submanifolds of $S_{1}^{6}$.

Q.E.D.

Added in proof. Recently Dr. Tasaki proved the following; Let $\mathfrak{l}$ and $\mathfrak{l}^{\prime}$ be semisimple Lie subalgebras of a compact semisimple Lie algebra $\mathfrak{g}$. If $\mathfrak{l}^{\boldsymbol{C}}$ and $\mathfrak{l}^{\prime} \boldsymbol{c}$ are conjugate in $\mathfrak{g}^{\boldsymbol{C}}$, then $\mathfrak{l}$ and $\mathfrak{l}^{\prime}$ are conkugate in $\mathfrak{g}$. Thus subalgebras in Lemma 3.9 , are conjugate in $g_{2}$.

\section{References}

[1] Bryant, R., Submanifolds and special structures on the octonions, J. Differential Geoncetry, 17 (1982), 185-232.

[2] Dynkin, E.B., Semi-simple subalgebras of semi-simple Lie algebras, A.M.S. Transl., Ser. 2, 6 (1957), 111-244.

[ 3 ] Ejiri, N., Totally real submanifold in the 6-sphere, Proc. A.M.S., 83 (1981), 759-763.

[4] Freudenthal, H., Oktaven, Ausnahmegruppen und Oktavengeometrie, Mimeographed Note, Utrecht, 1951.

[5] Harvey, R. and Lawson, H.B., Calibrated geometries, Acta Math., 148 (1982), 47-157.

[6] Hsiang, W.Y. and Lawson, H.B., Minimal submanifolds of low cohomogeneity, J. Differential Geometry, 5 (1971), 1-38.

[ 7 ] Mal'cev, A.I., On semi-simple subgroups of Lie groups, A.M.S. Transl, Ser. 1, 9 (1950), $172-213$.

[8] Siebenthal, J., Sur les sous-groupes fermes connexes d'un group de Lie clos, Coment. Math. Helv., 25 (1950), 210-256.

[9] Sugahara, K., The sectional curvature and the diameter estimate, Math. Japonica, 26 (1981), 153-159.

[10] Yano, K., The theory of Lie derivatives and its applications, North-Holland, Amsterdam, 1957.

Institute of Mathematics

University of Tsukuba

Sakura-muia, Niiharigun

Ibaraki, 305 Japan 